\title{
Surface plasmon polaritons on narrow-ridged short-pitch metal gratings
}

\author{
I. R. Hooper and J. R. Sambles \\ Thin Film Photonics Group, School of Physics, University of Exeter, Stocker Road, Exeter EX4 4QL, United Kingdom
}

(Received 20 May 2002; published 19 November 2002)

\begin{abstract}
The reflectivity of short pitch metal gratings consisting of a series of narrow Gaussian ridges in the classical mount has been modeled as a function of frequency and in-plane wave vector (the plane of incidence containing the grating vector) for various ridge heights. Surface plasmon polaritons (SPP's) are found to be excited even in the zero-order region of the spectrum. These may result in strong absorption of radiation polarized with its electric field in the plane of incidence (transverse magnetic). For zero in-plane wave vector the SPP modes consist of a symmetric charge distribution on either side of the grating ridges, a family of these modes existing with different numbers of field maxima per grating period. Because of the charge symmetry these modes may only be coupled to at angles away from normal incidence where strong resonant absorption may then occur. The dispersion of these SPP modes as a function of the in-plane wave vector is found to be complex arising from the formation of very large band gaps due to the harmonic content of the grating profile, the creation of a pseudo high-energy mode, and through strong interactions between different SPP bands.
\end{abstract}

DOI: 10.1103/PhysRevB.66.205408

PACS number(s): 78.20.-e

\section{INTRODUCTION}

Investigations into the optical response of metallic grating structures has recently enjoyed renewed interest. This is mainly due to their possible use as photonic band-gap structures, and the discovery of surface plasmon polariton (SPP) mediated enhanced transmission through arrays of subwavelength apertures. ${ }^{1-3}$ In addition, advances in fabrication techniques have allowed nano-structured surfaces with a designer optical response to be manufactured.

The surface plasmon polariton is a surface charge density oscillation at a metal/dielectric interface coupled to incident EM radiation with fields decaying exponentially away from the surface into each medium. ${ }^{4}$ These fields decay much faster into the metal than into the dielectric due to the different permittivities of the two media. By solving Maxwell's equations at a planar metal/dielectric boundary the dispersion relation of the flat-surface SPP may be obtained, and is given by

$$
k_{\mathrm{SPP}}=k_{0}\left(\frac{\varepsilon_{1} \varepsilon_{2}}{\varepsilon_{1}+\varepsilon_{2}}\right)^{1 / 2},
$$

where $k_{\mathrm{SPP}}$ is the wave vector of the SPP, $k_{0}$ is the wave vector of the incident EM radiation, and $\varepsilon_{1}$ and $\varepsilon_{2}$ are the permittivities of the dielectric and metal on either side of the interface, with $\left|\varepsilon_{2}\right|>\varepsilon_{1}$ and the real part of $\varepsilon_{2}<0$.

It is normal to plot this dispersion relation as a $\omega$ vs $k_{x}$ plot, where $\omega$ is the angular frequency and $k_{x}$ is the in-plane wave vector. Since $k_{x}=k_{0} \sin \theta$, where $\theta$ is the polar angle of incidence, the maximum $k_{x}$ which the incident radiation may have is at grazing incidence where $k_{x}=k_{0}$. From Eq. (1) it is clear that the wave vector of the SPP is always greater than the maximum $k_{x}$ available to the radiation, and therefore the SPP cannot be directly optically excited on such a planar system.

One method used to overcome the fact that the wave vector needed to excite the SPP is greater than that available to incident EM radiation is to periodically corrugate the surface with a grating structure. This has the effect of folding the SPP bands at the Brillouin zone boundaries produced by the periodicity (the Brillouin zone boundaries occur at $k_{x}$ $=n k_{g} / 2$, where $n$ is any integer, $k_{g}=2 \pi / \lambda_{g}$, and $\lambda_{g}$ is the grating pitch) into the region of $\omega-k_{x}$ space which is available to incident radiation. Appropriately polarized radiation may then excite one of the SPP branches and be absorbed.

When a shallow grating structure is added to the metal surface the SPP dispersion relation of Eq. (1) becomes

$$
k_{\mathrm{SPP}}=k_{0}\left(\frac{\varepsilon_{1} \varepsilon_{2}}{\varepsilon_{1}+\varepsilon_{2}}\right)^{1 / 2}=k_{0} \sin \theta \pm n k_{g} .
$$

However, this simple relation is only valid for very shallow structures where the grating does not perturb the SPP. For very deep gratings, where this is no longer the case, the situation is far more complex due to the formation of band gaps, and the dispersion relation of the SPP's may not be found analytically. Therefore, numerical modeling is needed to determine the dispersion relation of the SPP's, and the optical response of the structure.

Until the last few years the majority of work on metallic monogratings has related to low aspect ratio structures. However, due to recent advances in grating fabrication technology, short-pitch high-aspect ratio structures may now be manufactured, and these have shown bands of resonant optical absorption due to the excitation of standing wave SPP's which are very different to those found on lower aspect ratio structures. The SPP bands observed on gratings consisting of very deep $\left(d>\lambda_{g}\right)$ and narrow $\left(w \ll \lambda_{g}\right)$ grooves have been shown to be very flat-banded across all available wave vectors. ${ }^{5,6}$ Through modeling it has also been shown that it is possible to excite these flat-banded SPP's on zero-order gratings where SPP excitation would not be possible on lower aspect ratio structures. ${ }^{7-9}$ However, the resonant absorption bands are found to be very broad and shallow. These flatbanded SPP modes have antisymmetric charge distributions 


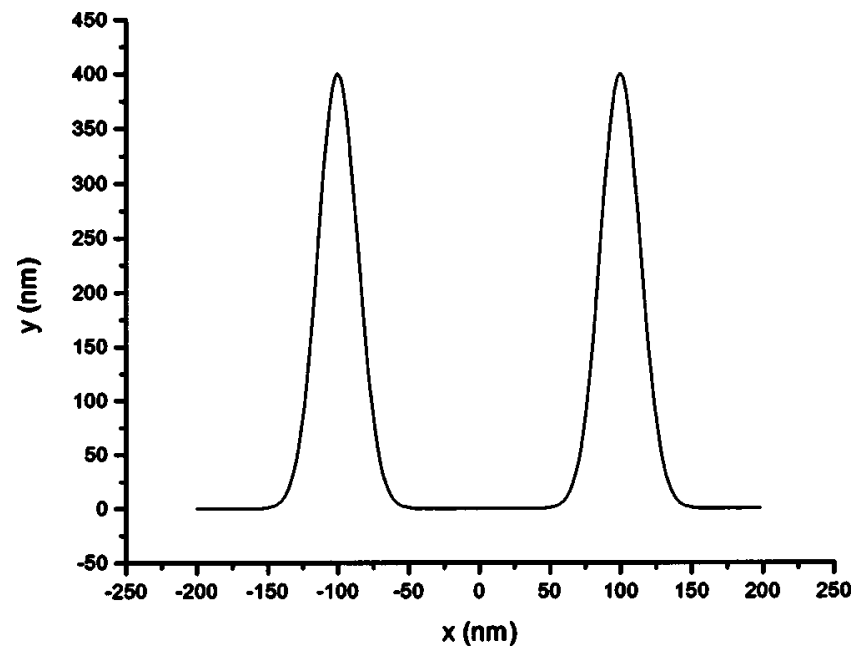

FIG. 1. An example of the type of grating structure investigated. The profile is made up of a series of Gaussian ridges defined by the grating pitch, ridge height, and ridge width (FWHM).

on either side of the grating grooves, the fields of which then couple together to produce a highly localized self-coupled SPP.

If the optical response of the inverse structure of high, narrow-ridged, gratings (Fig. 1) is calculated the dispersion of the SPP's are no longer found to be simple flat bands. However, strong absorption bands are found in the zero order region of the spectrum (Fig. 2). The time averaged fields calculated for the SPP mode on the reflectivity plot of Fig. 2 at $2 k_{x} / k_{g}=0.1$, and $f=0.88 \times 10^{15} \mathrm{~Hz}$ (shown in Fig. 3) shows that the SPP modes on narrow ridged gratings arise from a symmetric distribution of charges on either side of the grating ridges. It is these modes which we investigate in this paper.

\section{THE MODELING CODE}

The method used in the computer code for the modeling in this paper is based upon a method originally proposed by
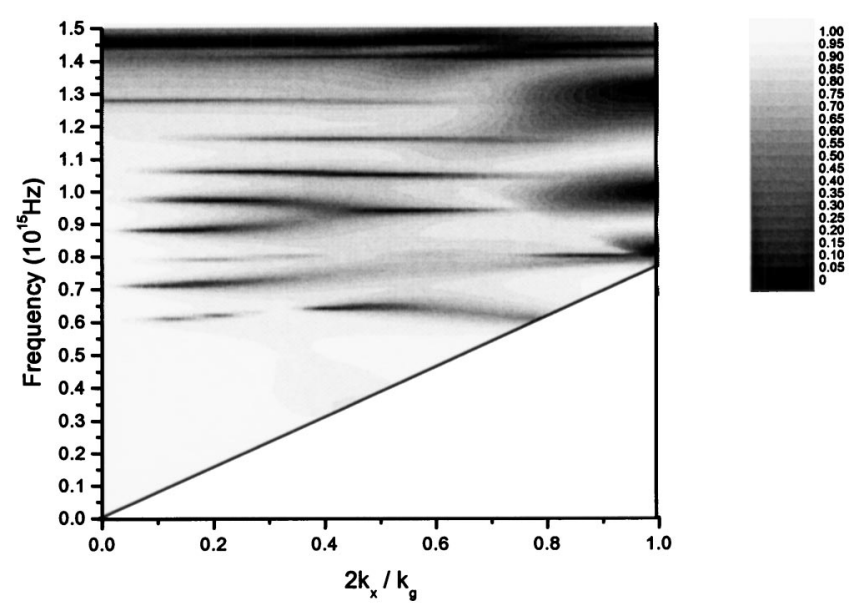

FIG. 2. TM reflectivity as a function of frequency and in-plane wave vector for a 200-nm-pitch silver grating consisting of a series of 400-nm-high and 40-nm-wide (FWHM) Gaussian ridges with light incident at a $0^{\circ}$ azimuthal angle.
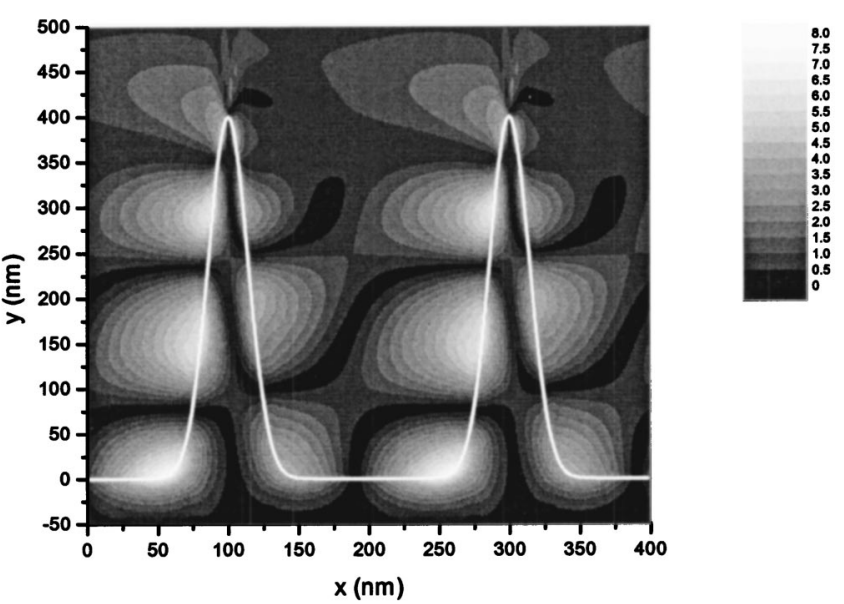

FIG. 3. Time averaged $H_{z}$ component of the fields of the SPP mode excited on a 200-nm-pitch silver grating consisting of a series of 400-nm-high and 40-nm-wide (FWHM) Gaussian ridges, with radiation of $f=0.88 \times 10^{15} \mathrm{~Hz}$ incident at a $0^{\circ}$ azimuthal angle and at $2 k_{x} / k_{g}=0.1$.

Chandezon et al., ${ }^{10}$ in which a nonorthogonal curvilinear coordinate transformation is used to map the grating profile onto a flat plane. This enables easier matching of the tangential components of the fields at the boundaries, from which a scattering matrix is determined. From this the fields in the two media are determined, and the reflection coefficients are calculated. It is also possible to identify the dispersion of any surface modes by identifying poles in the scattering matrix. This enables the dispersion of the modes to be obtained in regions of $\omega-k_{x}$ space which would not be accessible through investigating the reflectivity from a surface. This is because the scattering matrix does not depend upon the coupling of the surface mode to incident radiation. The method used in this code has been reported in more detail elsewhere. $^{11}$

In all of the following work the permittivity of the metal gratings is modeled as that of silver, being described by a Drude model with a plasma frequency of $\omega_{p}=1.32$ $\times 10^{16} \mathrm{~s}^{-1}$, and a relaxation time of $\tau=1.45 \times 10^{-14} \mathrm{~s}$.

\section{BAND GAPS AND THE FORMATION OF SPP'S ON NARROW-RIDGED SHORT-PITCH GRATINGS}

When a sinusoidal grating is irradiated at normal incidence with EM radiation of the correct frequency and polarization state to excite a SPP, what is actually excited are two SPP's, propagating in the $+x$ and $-x$ directions, respectively. These interact to produce a standing wave which may have two possible field configurations relative to the grating profile, one with $H$-field maxima on the sides of the grating ridges, and one with $H$-field maxima at the ridges and troughs of the grating profile. Due to their different field distributions these two standing waves have different energies, and therefore a band gap is opened. ${ }^{12,13}$ The two solutions also correspond to symmetric and antisymmetric charge distributions on either side of the grating ridges. At normal incidence it is impossible for incident radiation to couple to 
(a)

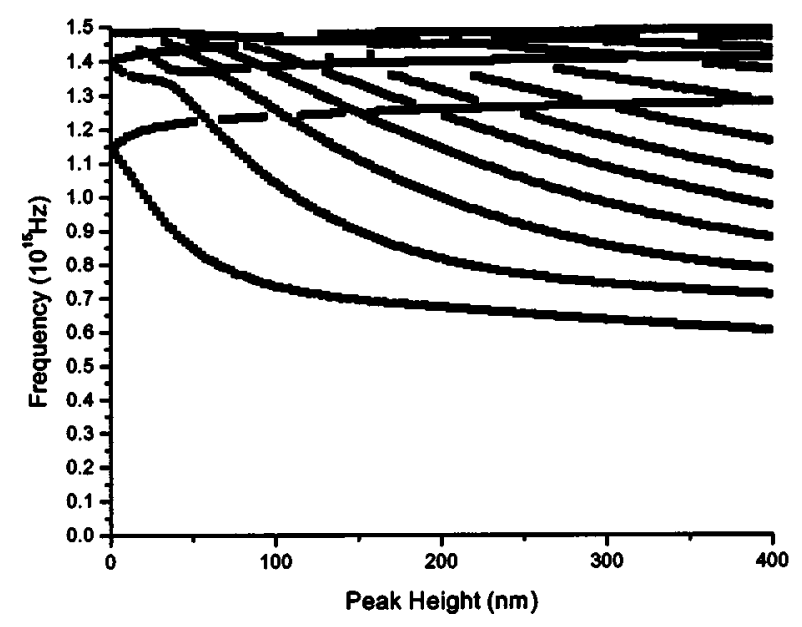

(b)

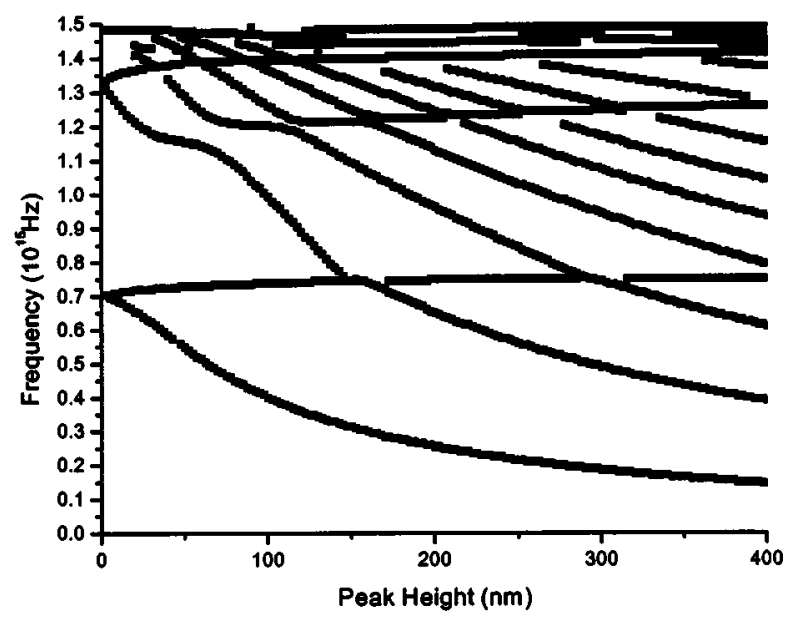

FIG. 4. Mode frequency as a function of grating ridge height for a 200-nm-pitch grating consisting of 40-nm-wide (FWHM) Gaussian ridges at a $0^{\circ}$ azimuthal angle and (a) $2 k_{x} / k_{g}=0$ and (b) $2 k_{x} / k_{g}=1.0$.

the symmetric charge distribution case since it would require the incident radiation $E$ fields to point in opposite directions on either side of the grating groove at the same instant in time. This, of course, is not possible for a normally incident plane wave. However, if the radiation is incident at some polar angle $\theta$ then the mode may be excited.

When the grating profile is more complicated than a pure sinusoid it is the details of the grating shape that determines which of these solutions is the high energy and which is the low energy. If the grating profile is described by a finite Fourier series of the form

$$
f(x)=a_{0} \sin \left(k_{g} x+\phi_{1}\right)+a_{1} \sin \left(2 k_{g} x+\phi_{2}\right)+\cdots,
$$

then, for the first order SPP at normal incidence (corresponding to scattering from $\pm k_{g}$ ), it is the $2 k_{g}$ component which couples together the two counterpropagating SPP's. Therefore it is this component which predominantly determines the size of this band gap, and also which of the two solutions is the higher in energy. The high-energy solution is the mode which has $H$-field maxima at the ridges of the $2 k_{g}$ component of the grating profile, and the low-energy solution is that with $H$-field maxima at the troughs of the $2 k_{g}$ component. Therefore, if the phase between the $k_{g}$ and the $2 k_{g}$ components is $+90^{\circ}$, the low-energy branch consists of $H$-field maxima at the ridges and troughs of the grating since these are the positions at which the $2 k_{g}$ component has its troughs (which correspond to an antisymmetric charge distribution on either side of the grating grooves). This branch may then be coupled to at normal incidence, whereas the high-energy branch may not since the $H$-field maxima of this mode occur at the shoulders of the grating grooves (which correspond to a symmetric charge distribution). If the phase between them is $-90^{\circ}$, the result is reversed, and the upper energy branch is coupled to at normal incidence, and not the lower-energy branch. The Gaussian ridged profile investigated here is an example of this second case.

For other SPP branch crossing points, whether at the Brillouin zone boundary or at normal incidence, the process is very similar. The component of the grating profile which couples together the two counterpropagating SPP's, and therefore the one that produces the band gap, is always the sum of the two SPP scattering processes involved in creating the SPP at this point. For example, the second-order SPP branch crossing point at $2 k_{x}=k_{g}$ arises through scattering from $-k_{g}$ and $+2 k_{g}$, and therefore it is the $3 k_{g}$ component of the grating profile which produces the band gap.

Another point to note is that the number of field maxima per grating period for the SPP is determined by the scattering processes involved. For the first order SPP at the Brillouin zone boundary there is one field maximum per grating period. The first-order SPP at normal incidence has two field maxima, since it arises from scattering by $\pm k_{g}$, and the second order SPP at the Brillouin zone boundary has three maxima since it arises from scattering by $-k_{g}$ and $+2 k_{g}$. Therefore, SPP's at the Brillouin zone boundary have charge distributions which are not the same for each grating period, but which repeat alternately due to the fact that they have an odd number of field maxima per grating period.

For the narrow-ridged grating profiles investigated in this paper the phase of the odd integer $k_{g}$ components of the grating profile is always $0^{\circ}$. Therefore, the low-energy branches of band gaps arising at the Brillouin zone boundary have $H$-field maxima on the grating ridge sides, and also at the midpoint between grating ridges.

For relatively shallow gratings $\left(d \ll \lambda_{g}\right)$ the formation of band gaps is well understood. However, when the grating height is increased so that it is of the order of the grating pitch the situation becomes more complex. The mode frequency, for $k_{x}=0$, as a function of grating ridge height for a 200-nm pitch grating consisting of a series of 40-nm-wide Gaussian ridges at a $0^{\circ}$ azimuthal angle is shown in Fig. 4(a). For small ridge heights a band gap corresponding to the firstorder SPP opens at $f \approx 1.14 \times 10^{15} \mathrm{~Hz}$. The low-energy branch rapidly reduces in energy with increasing ridge height, whereas the high-energy branch is observed to increase in energy relatively little. The reason for this asym- 
(a)

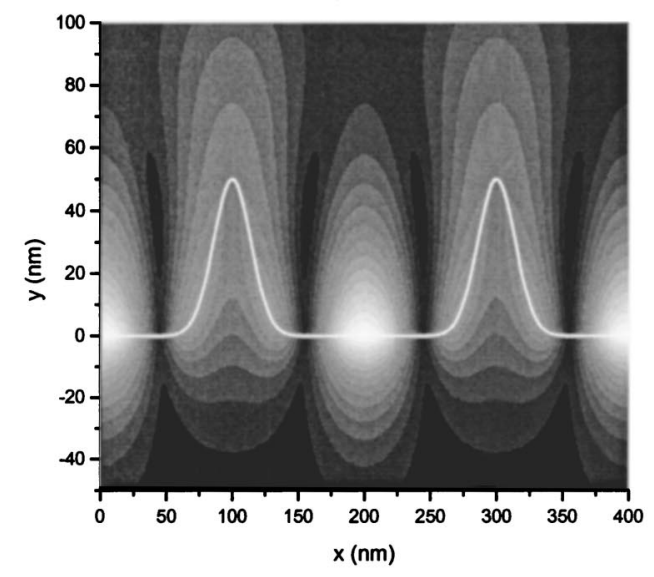

(b)

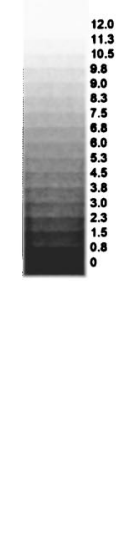

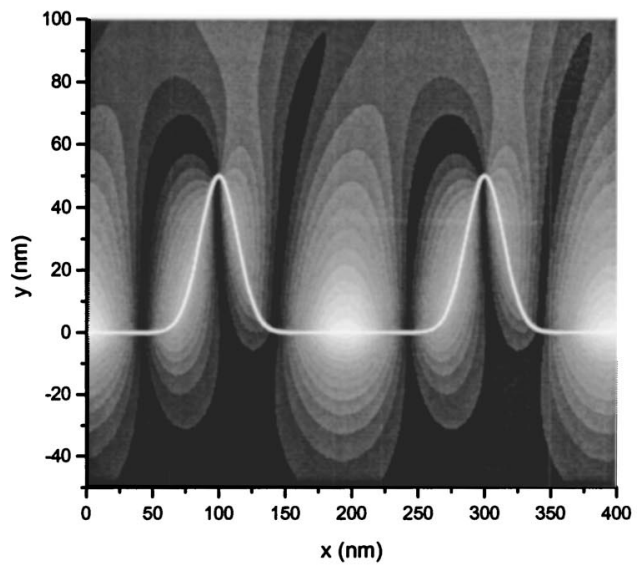

FIG. 5. Time averaged $H_{z}$ component of the fields of SPP modes excited on a 200-nm-pitch silver grating consisting of a series of 50-nm-high and 40-nm-wide (FWHM) Gaussian ridges at a $0^{\circ}$ azimuthal angle. (a) $f=1.22 \times 10^{15} \mathrm{~Hz}$ and $2 k_{x} / k_{g}=0$ and (b) $f=1.16$ $\times 10^{15} \mathrm{~Hz}$ and $2 k_{x}=k_{g}=1.0$.

metric splitting is that the high-energy branch may only rise in energy to asymptotically approach its associated diffraction edge, whereas the low-energy branch has no such constraint.

As the ridge heights increase low-energy branches of higher-order SPP's are observed to reduce in energy through the high-energy branch of the first-order SPP. Also, the lowenergy branches appear to have a limit to the energy they may reduce to of $f \approx 0.6 \times 10^{15} \mathrm{~Hz}$. However, this is not actually the case and this apparent limit arises at the frequency at which the lowest band is flat across the whole of the Brillouin zone. In fact, this rapid reduction in the rate at which the low-energy branches lower in frequency occurs at any point where a flat band has formed across the entire Brillouin zone.

This behavior can best be observed in the plots of the mode frequency as a function of ridge height at the BZ boundary [Fig. 4(b)]. In this plot there appears to be anticrossing of two modes at a frequency of between 1.1-1.2 $\times 10^{15} \mathrm{~Hz}$, although in fact there is no mode starting at $f$ $=1.1 \times 10^{15} \mathrm{~Hz}$. However, this frequency is very close to that of the high-energy mode at $k_{x}=0$. The change in energy of this apparent anticrossing as a function of ridge height clearly follows that of the high-energy mode at $k_{x}=0$. This implies that when a low-energy branch at either symmetry point occurs at the same frequency as a high-energy branch at the other symmetry point (in other words, when the band is flat across the whole of the Brillouin zone) then the lowenergy branch follows the dispersion of the high-energy mode as a function of ridge height until the next higher-order low-energy SPP branch anticrosses with it. (In other words it behaves as a pseudo high-energy mode.)

Some understanding of this behavior can be gained by investigating the field distributions for the high-energy SPP branches at $k_{x}=0$, and the low-energy SPP branches at $2 k_{x}$ $=k_{g}$ which occur at close to the same frequency. For a grating pitch of $200 \mathrm{~nm}$ the first grating ridge height at which this occurs is at $d=50 \mathrm{~nm}$, and the time averaged $H_{z}$ component of the fields for the mode at near-normal incidence, together with that at the Brillouin zone boundary are shown in Fig. 5. The obvious difference between these is that a maximum in the $H_{z}$ distribution is evident on the tops of the grating ridges for the SPP at near-normal incidence, which is absent in the field distribution for the mode at the Brillouin zone boundary. However, in both cases it is clear that the strongest fields are those which occur at the grating troughs.

Since the dispersion in frequency as a function of grating ridge height at $d \approx 50 \mathrm{~nm}$ is strongly affected by the secondorder low-energy mode this is far clearer for larger grating ridge heights where the anticrossing at the Brillouin zone boundary between this pseudo high-energy mode and the higher-order low-energy modes is less pronounced. In Fig. 6 the time averaged $H_{z}$ component of the fields are shown for $d=350 \mathrm{~nm}$ for the pseudo-high-energy mode at the Brillouin zone boundary. Again, the strongest fields are found on the flat portion within the grating troughs, with the fields on the grating ridges being of a similar magnitude to the interfer-
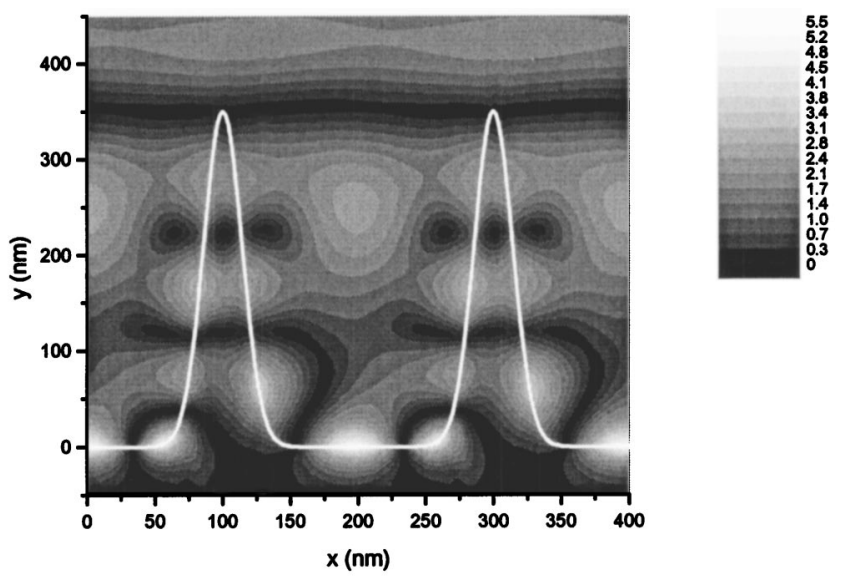

FIG. 6. Time averaged $H_{z}$ component of the fields of the SPP mode excited on a 200-nm-pitch silver grating consisting of a series of 350-nm-high and 40-nm-wide (FWHM) Gaussian ridges, with radiation of $f=1.26 \times 10^{15} \mathrm{~Hz}$ incident at a $0^{\circ}$ azimuthal angle and at $2 k_{x} / k_{g}=1.0$. 
(a)

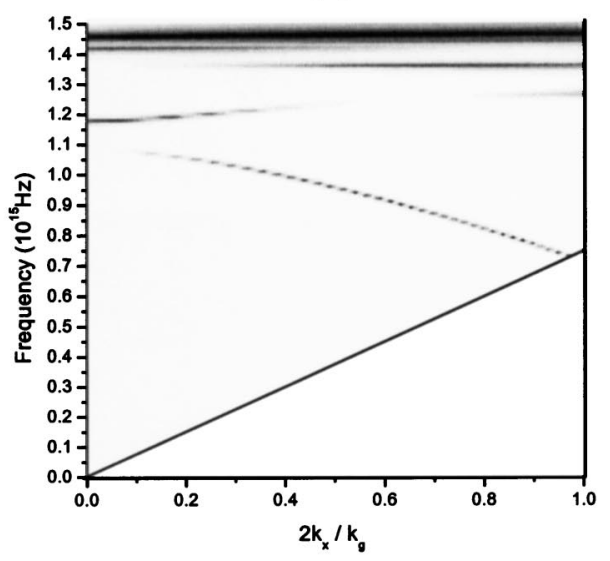

(c)

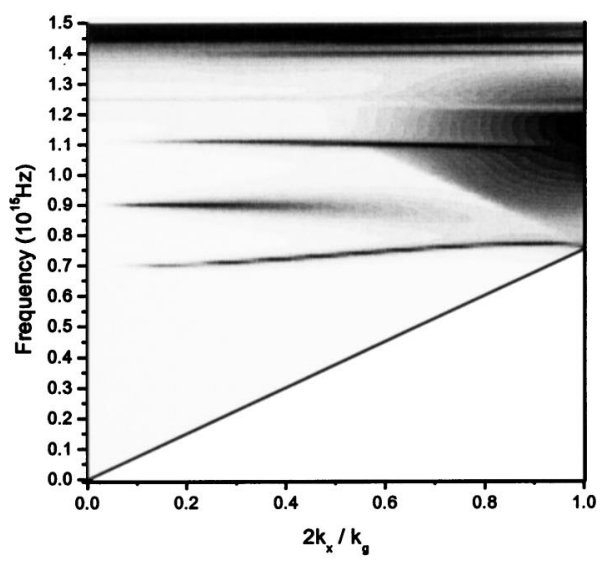

(b)
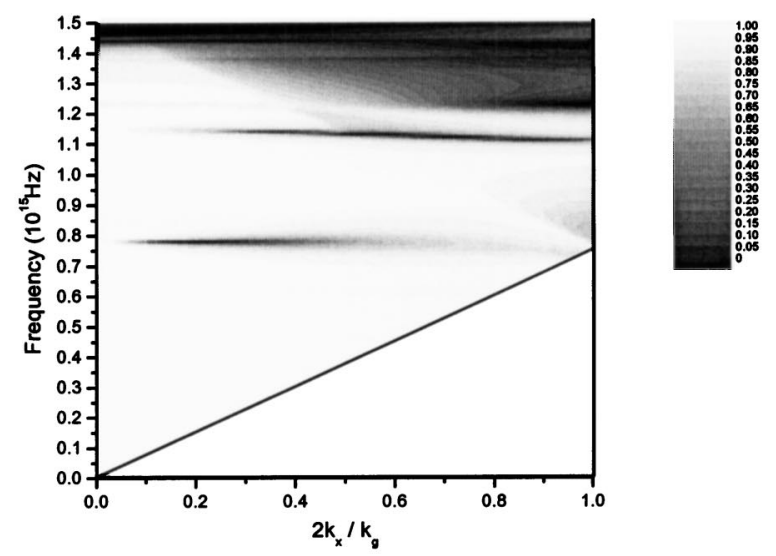

(d)

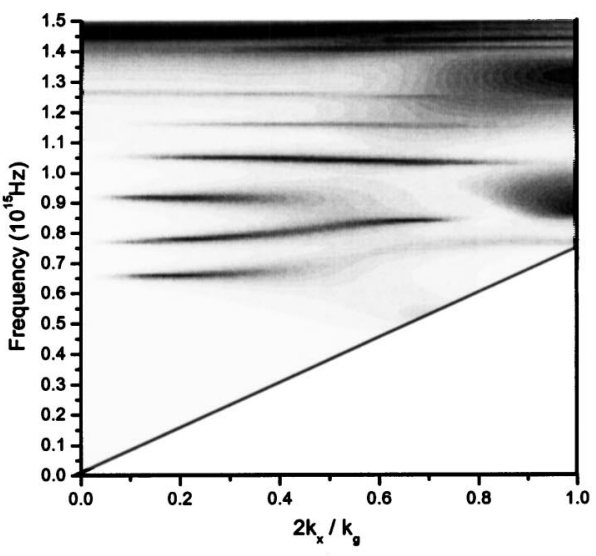

FIG. 7. TM reflectivity as a function of frequency and in-plane wave vector for a 200-nm-pitch silver grating, consisting of a series of 40-nm-wide (FWHM) Gaussian ridges and ridge heights of (a) $10 \mathrm{~nm}$, (b) $75 \mathrm{~nm}$, (c) $150 \mathrm{~nm}$, and (d) $250 \mathrm{~nm}$.

ence oscillation arising from the incident and reflected waves. In fact the spatial distribution of the strong fields in the grating with $d=350 \mathrm{~nm}$ have the same periodicity as those with $d=50 \mathrm{~nm}$. Therefore, we believe that the pseudohigh-energy mode at $f \approx 1.2 \times 10^{15} \mathrm{~Hz}$ at the Brillouin-zone boundary arises from a first-order low-energy mode, which then anticrosses with the higher-order low-energy modes as they lower in frequency with increasing grating ridge height. The additional field maxima on the ridges for the field distribution with $d=350 \mathrm{~nm}$ arise due to the mixed character of the mode caused by the anticrossing with the higher-order low-energy modes.

Similarly, the apparent low-energy limit of the lowestenergy SPP branch at normal incidence is also a frequency at which a pseudo high-energy mode occurs. In this case the first-order low-energy SPP branch at $k_{x}=0$ is almost flat with the first-order high-energy branch at the Brillouin zone boundary, and at this point the fields of the two modes are similar in the same way as described above. The apparent anticrossing is far less pronounced in this case since the lowenergy modes are reducing in energy relatively slowly as they reach the frequency at which the anticrossing takes place. Therefore it is not particularly clear for the grating height range investigated here, which is limited by the convergence limits of the modeling code. However, we believe that for larger grating ridge heights these modes could be excited at lower frequencies.

From Fig. 4 the dispersion of these modes at normal incidence and at $2 k_{x} / k_{g}=1$ are well defined. However, these do not explain the complex dispersion behavior shown in the reflectivity plot of Fig. 2. In order to gain some insight into how this complex dispersion with changing in-plane wave vector arises it is useful to investigate the TM reflectivities as a function of both frequency and in-plane wave vector for different heights of the grating ridges. These are shown in Fig. 7. Figure 7(a) shows the reflectivity from a $200-\mathrm{nm}$ pitch silver grating consisting of a series of 40-nm-wide, 10-nm-high, Gaussian ridges. This clearly shows the band gap at $k_{x}=0$ opened due to the large first harmonic component inherent in the Gaussian ridged profile.

In Fig. 7(b) the Gaussian ridge heights have been increased to $75 \mathrm{~nm}$. The first-order low-energy branch at $k_{x}$ $=0$ has lowered in frequency sufficiently to produce a relatively flat band with the first-order high-energy branch at the Brillouin zone boundary. In addition the second-order lowenergy branch at normal incidence has anticrossed with the 
first-order high-energy branch and has produced a band at $f \approx 1.13 \times 10^{15} \mathrm{~Hz}$.

It would be expected on such a grating that the first-order SPP mode at normal incidence would produce a very broad and shallow reflectivity minimum since the radiative damping of a mode is known to increase with increasing depth/ height of a grating. However, because these modes have fields which extend further into the metal than is the case with gratings consisting of narrow grooves (where the charge distribution is antisymmetric on either side of the grating grooves), the resistive damping is also high. Since the resonance shape depends upon the ratio of the resistive and radiative damping components, with a reflectivity of zero when the two components are equal, this increase in the resistive damping compensates for the increase in the radiative damping. Therefore a deep reflectivity minimum is observed even on a high aspect ratio grating.

When the height of the ridges is increased to $150 \mathrm{~nm}$ [Fig. 7 (c)] significant changes to the mode dispersions have occurred. The first-order branch has lowered in energy very little since it is almost flat across the entire Brillouin zone. However, the end of the second-order SPP branch at the Brillouin zone boundary now coincides with that of the firstorder branch. Due to the fact that the modes may disperse in frequency as a function of grating height more rapidly at the Brillouin zone boundary than at normal incidence it is now closer in frequency to the first-order SPP at normal incidence. Therefore the first-order SPP has coupled more strongly to it leaving only weak coupling with the secondorder SPP at $k_{x}=0$.

In Fig. 7(d) the grating ridge height has been increased to $250 \mathrm{~nm}$. The dispersion of the modes has developed from the 150 -nm case in that the lowest-energy mode now weakly couples to a point on the lightline at $f \approx 0.52 \times 10^{15} \mathrm{~Hz}$, as well as the first high-energy mode at the Brillouin zone boundary. In Fig. 4(b) these nonradiative modes are evident below the lightline, which occurs at $f=0.75 \times 10^{15} \mathrm{~Hz}$. The second order of these modes occurs at $f \approx 0.5 \times 10^{15} \mathrm{~Hz}$ and is an almost flat band across to the lightline. This mode shows very weak coupling to radiation inside of the lightline since the branch of which it consisted before it become nonradiative remains coupled to the first-order high-energy mode at the Brillouin zone boundary.

The remaining band structure within the Brillouin zone can best be understood by considering which of the bands at normal incidence couple to which at the Brillouin zone boundary. This is simply achieved by comparing Fig. 7(d) with those of Fig. 4.

In this we define LE and HE to refer to the low- and high-energy modes, respectively, followed by an integer which defines the order of the mode. From Figs. 4 and 7(d) it can be seen that LE1 at normal incidence links most strongly with HE1 at the Brillouin zone boundary, and LE2 links most strongly with LE3. Since LE4 at normal incidence is strongly coupled to LE4 at the Brillouin zone boundary after anti- crossing with the HE2 band, this leaves no branch at the Brillouin zone boundary for the LE3 branch at normal incidence to link to. Therefore it is observed to join only weakly with the LE3 band at the Brillouin zone boundary. For higher grating ridge heights the number of SPP branches at normal incidence which only have weak linkage to the branches at the Brillouin zone boundary is increased, as is evident in Fig. 2. This is because the dispersion in frequency of the SPP modes at the Brillouin zone boundary as a function of grating ridge height is more rapid, and therefore a higher number of SPP modes at the Brillouin zone boundary are at a lower frequency than the first-order SPP at normal incidence.

(Note that, as the grating ridge height is increased, one eventually reaches the point where the radius of curvature of the grating ridges is less than the electron mean free path or the skin depth, and therefore the use of a simple local dielectric function for the metal will become invalid. Therefore, the modeling for the gratings consisting of high ridges in this paper may be flawed in these cases, however, we believe that though this may alter the coupling to the modes it will not significantly change their dispersion.)

\section{CONCLUSIONS}

A series of SPP resonances have been shown to occur on metal gratings consisting of high and narrow Gaussian ridges in the zero-order region of the spectrum. At near-normal incidence these SPP's consist of a symmetric charge distribution on either side of a grating ridge coupled to TM polarized radiation. They may not be excited at normal incidence due to this charge distribution, but for incident angles away from normal incidence strong absorption may occur. The dispersion of these SPP modes in frequency as a function of inplane wave vector is complex, and is determined by the very large band gaps produced by the large harmonic content of the Gaussian ridged profile, the creation of a pseudo-highenergy mode, and through interactions between SPP bands.

The situation is very different to that found for narrow Gaussian grooved gratings where broad, very flat-banded, standing wave SPP modes are excited. For the Gaussian ridged case the reflectivity minima are sharper and much less flat banded. The SPP modes also have a very different character, and the interactions between the different SPP bands as the ridge height is increased is different to that of the Gaussian grooved structures. This change in going from Gaussian grooves to Gaussian ridges further highlights the potential for high aspect ratio zero-order metal grating surfaces with specific absorption properties.

\section{ACKNOWLEDGMENTS}

The authors are grateful for EPSRC support and the provision of a CASE award fromQinetiQ (Farnborough) for I. R. H. This work was carried out as part of Technology Group 08 of the MoD Corporate Research Fund. 
${ }^{1}$ T. W. Ebbesen, H. J. Lezec, H. F. Ghaemi, T. Thio, and P. A. Wolff, Nature (London) 391, 667 (1998).

${ }^{2}$ H. F. Ghaemi, Tineke Thio, D. E. Grupp, T. W. Ebbesen, and H. J. Lezec, Phys. Rev. B 58, 6779 (1998).

${ }^{3}$ L. Martin-Moreno, F. J. Garcia-Vidal, H. J. Lezec, K. M. Pellerin, T. Thio, J. B. Pendry, and T. W. Ebbesen, Phys. Rev. Lett. 86, 1114 (2001).

${ }^{4}$ H. Raether, Surface Plasmons (Springer-Verlag, Berlin, 1988).

${ }^{5}$ T. Lopez-Rios, D. Mendoza, F. J. Garcia-Vidal, J. SanchezDehesa, and B. Pannetier, Phys. Rev. Lett. 81, 665 (1998).

${ }^{6}$ F. J. Garcia-Vidal, J. Sanchez-Dehesa, A. Dechelette, E. Bustarret, T. Lopez-Rios, T. Fournier, and B. Pannetier, J. Lightwave Technol. 17, 2191 (1999).
${ }^{7}$ M. B. Sobnack, W. C. Tan, N. P. Wanstall, T. W. Preist, and J. R. Sambles, Phys. Rev. Lett. 80, 5667 (1998).

${ }^{8}$ W. C. Tan, T. W. Preist, J. R. Sambles, and N. P. Wanstall, Phys. Rev. B 59, 12661 (1999).

${ }^{9}$ I. R. Hooper, and J. R. Sambles, Phys. Rev. B 65, 165432 (2002).

${ }^{10}$ J. Chandezon, M. T. Dupuis, G. Cornnet, and D. Maystre, J. Opt. Soc. Am. 72, 839 (1982).

${ }^{11}$ N. P. K. Cotter, T. W. Preist, and J. R. Sambles, J. Opt. Soc. Am. A 12, 1097 (1995).

${ }^{12}$ W. L. Barnes, T. W. Preist, S. C. Kitson, J. R. Sambles, N. P. Cotter, and D. J. Nash, Phys. Rev. B 51, 11164 (1995).

${ }^{13}$ W. L. Barnes, T. W. Preist, S. C. Kitson, and J. R. Sambles, Phys. Rev. B 54, 6227 (1996). 